\title{
JORDAN HOMOMORPHISMS AND RIGHT ALTERNATIVE RINGS
}

\author{
M. F. SMILEY
}

Introduction. An additive mapping $a \rightarrow a^{\prime}$ of an associative ring $R$ into an associative ring $R^{\prime}$ is a Jordan homomorphism as defined by Jacobson and Rickart [2] in case

$$
\begin{aligned}
\left(a^{2}\right)^{\prime} & =\left(a^{\prime}\right)^{2}, \\
(a b \cdot a)^{\prime} & =a^{\prime} b^{\prime} a^{\prime}
\end{aligned}
$$

for every $a, b \in R$. R. L. San Soucie [4] calls a nonassociative ring $R$ strongly right alternative in case its right multiplications $a^{\prime}: x \rightarrow x a$ satisfy (I) and (II). (Every right alternative ring in which $2 a=0$ implies $a=0$ is strongly right alternative just as (I) implies (II) under the same assumption in the associative case.) In a recent paper [5] we developed some identities for Jordan homomorphisms in the associative case which were extensions of those previously given. (See, especially, I. N. Herstein [1].) It turns out that the nonassociative analogues of these identities are useful in reducing strongly right alternative rings to alternative rings. To do this one invokes the property

$\left(\mathrm{P}_{0}\right)$ If $x, y, z \in R$ and $(x, y, z)^{2}=0$, then $(x, y, z)=0$ provided $x$ has one of the forms $y,[y, z],[y, z] y,(y, y, z)$ or provided $z=w y$ and $x=(y, y, w)$.

(Property $\left(\mathrm{P}_{0}\right)$ is the operational form of E. Kleinfeld's Property (P) [3].) We give in this note a proof that (I), (II) and $\left(\mathrm{P}_{0}\right)$ imply that $R$ is an alternative ring. This result subsumes those of Kleinfeld [3] and of San Soucie [4] in this connection. Our main interest is not in the slightly greater generality of this result but rather in the method of proof. The reader will find, we hope, that our proof is straightforward and relatively brief. We have made our presentation self-contained but we have relegated some simple computations, most of which are strictly analogous to those given by us in [5], to an Appendix.

1. Identities. We linearize (I) and (II) to obtain

$$
(a b+b a)^{\prime}=a^{\prime} b^{\prime}+b^{\prime} a^{\prime}, \quad(a b \cdot c+c b \cdot a)^{\prime}=a^{\prime} b^{\prime} c^{\prime}+c^{\prime} b^{\prime} a^{\prime},
$$

for every $a, b, c \in R$. We adopt the abbreviations

Presented to the Society, April 20, 1957; received by the editors October 29, 1956. 


$$
a^{b}=(a b)^{\prime}-a^{\prime} b^{\prime}, \quad a_{b}=(a b)^{\prime}-b^{\prime} a^{\prime}
$$

of Herstein [1] and note that

$$
\begin{aligned}
a^{b}+b^{a} & =a_{b}+b_{a}=0, & a_{b}+a^{b} & =[a, b]^{\prime}, \\
x a^{b} & =-(x, a, b), & x a_{b} & =x[a, b]+(x, a, b)
\end{aligned}
$$

for every $x, a, b \in R$. With these abbreviations, we compute

$$
a^{b} a_{b}=0, \quad a_{b} a^{b}=-([a, b], a, b)^{\prime}
$$

for every $a, b \in R$. From (II) and (2) we find that

$$
a^{a b}=-(a b)^{a}=a^{b} a^{\prime}, \quad a_{a b}=-(a b)_{a}=a^{\prime} a_{b}
$$

for every $a, b \in R$. By direct computation (given in the Appendix) we obtain

$$
\begin{gathered}
a^{b} a^{\prime} a_{b}=0, \\
(a, a, b)^{\prime}=a_{b a}-a_{b} a^{\prime}=a^{b a}-a^{\prime} a^{b}=-a^{q}-a^{\prime} a^{b}+a^{b} a^{\prime}
\end{gathered}
$$

with $q=[a, b]$ for every $a, b \in R$. Using (6), (4), (3), and (5), we see that

$$
a b+b a=0 \text { for } a, b \in R \text { implies that } a^{b}(a, a, b)^{\prime}=0 .
$$

Again using the computations of the associative case [5], we obtain

$$
\begin{aligned}
a^{b} r^{\prime}+r^{\prime} a_{b} & =\left(r a_{b}\right)^{\prime}, \\
r^{\prime} a^{b}+a_{b} r^{\prime} & =([a, b] r-(r, a, b))^{\prime}
\end{aligned}
$$

for every $a, b, r \in R$. Replacing $r$ in (8) by $(r, a, b)=-r a^{b}$ we see, by (3), that

$$
a^{b}(r, a, b)^{\prime}+(r, a, b)^{\prime} a_{b}=0
$$

for every $a, b, r \in R$. Manipulations of (8), as in [5], then yield

$$
a_{b} r^{\prime} a^{b}+a^{b} r^{\prime} a_{b}+([a, b] r, a, b)^{\prime}=0
$$

for every $a, b, r \in R$. (It was the associative analogue of (10) which was decisive in [5].) Setting $r=a$ in (10) gives, by (5) and (3),

$$
a_{b} a^{\prime} a^{b}+([a, b] a, a, b)^{\prime}=0, \quad a^{b}([a, b] a, a, b)^{\prime}=0
$$

for every $a, b \in R$.

2. Proof of the theorems of Kleinfeld and of San Soucie. We now assume $\left(\mathrm{P}_{0}\right)$ as well as (I) and (II), and we shall prove that $R$ is an alternative ring. For $a, b \in R$, we adopt the notation of Kleinfeld: 
$p=(a, a, b), q=[a, b]$. Then $\left(\mathrm{P}_{0}\right),(3)$, and (11) give $a_{b} a^{b}=(q, a, b)$ $=a_{b} a^{\prime} a^{b}=(q a, a, b)=0$. Using (6), we have $q p=q p^{\prime}=-q a^{q}=(q, a, q)$ $=q a^{b a}$; and, by a linearization of (3), $q p^{2}=q a^{b a}\left(a_{b a}-a_{b} a^{\prime}\right)=-q a^{b a} a_{b} a^{\prime}$ $=q a^{b} a_{b a} a^{\prime}=0$. Next, using (3), $(a q, a, b)=-a q^{\prime} a^{b}=-a\left(a^{b}\right)^{2}=-(p, a, b)$ $=p q$. Linearizing $(q, a, b)=0$ gives $([a, q], a, b)+(q, a, q)=0$, i.e., $p q+q p=0$. By $(7)$ and $\left(\mathrm{P}_{0}\right),(p, p, q)=(q, q, p)=0$. Then $p^{2} q=p(p q)$ $=-p(q p)=-(p q) p=(q p) p=0$. Since, by $(4),(q, p, p q)=(q, p, q) p$ $=0$, we see that $(q p)(p q)=-(p q)^{2}=0$, and $\left(\mathrm{P}_{0}\right)$ yields $p q=0=(p, a, b)$ $=a\left(a^{b}\right)^{2}$. Linearizing, we get $a a^{b} a^{a b}+a a^{a b} a^{b}=0$, and (4) gives $a a^{b} a^{\prime} a^{b}$ $=0$. Hence $p^{2}=-a a^{b} p^{\prime}=-a a^{b} a^{b a}=-(p, a, b a), p^{3}=-a a^{b} a^{b a}\left(a_{b a}\right.$ $\left.-a_{b} a^{\prime}\right)=a a^{b} a^{b a} a_{b} a^{\prime}=-a\left(a^{b}\right)^{2} a_{b a} a^{\prime}=0$. By $\left(\mathrm{P}_{0}\right), p^{2}=0$; and $p=0$ by $\left(\mathrm{P}_{0}\right)$. This completes the proof.

\section{Appendix}

Proof of (2). The first two equations restate the first equation of (1), while $[a, b]^{\prime}=(a b-b a)^{\prime}=a^{\prime} b^{\prime}+b^{\prime} a^{\prime}-2(b a)^{\prime}=-b^{a}-b_{a}=a^{b}+a_{b}$, $x a^{b}=x(a b)-(x a) b=-(x, a, b)=x[a, b]-x a_{b}$.

ProOF OF (3). We compute

$$
\begin{aligned}
a^{b} a_{b} & =(a b)^{\prime}(a b)^{\prime}+a^{\prime}\left(b^{\prime}\right)^{2} a^{\prime}-a^{\prime} b^{\prime}(a b)^{\prime}-(a b)^{\prime} b^{\prime} a^{\prime} \\
& =\left((a b)(a b)+\left(a b^{2}\right) a-(a b)(a b)-\left(a b^{2}\right) a\right)^{\prime}=0,
\end{aligned}
$$

while

$$
\begin{aligned}
a_{b} a^{b} & =(a b)^{\prime}(a b)^{\prime}+b^{\prime}\left(a^{\prime}\right)^{2} b^{\prime}-b^{\prime} a^{\prime}(a b)^{\prime}-(a b)^{\prime} a^{\prime} b^{\prime} \\
& =\left((a b)(a b)+\left(b a^{2}\right) b-(b a)(a b)-(a b \cdot a) b\right)^{\prime} \\
& =-((a b-b a), a, b)^{\prime} .
\end{aligned}
$$

Proof of (5). We have

$$
a^{b} a^{\prime} a_{b}=(a b)^{\prime} a^{\prime}(a b)^{\prime}-(a b)^{\prime} a^{\prime} b^{\prime} a^{\prime}-a^{\prime} b^{\prime} a^{\prime}(a b)^{\prime}+a^{\prime} b^{\prime} a^{\prime} b^{\prime \prime} a .
$$

Using (II) and (1),

$$
\begin{aligned}
a^{b} a^{\prime} a_{b} & =\{(a b \cdot a)(a b)-(a b)(a b \cdot a)-(a b \cdot a)(a b)+(a(b a \cdot b)) a\} \\
& =\left(a b^{\prime} a^{\prime} b^{\prime} a^{\prime}-a b^{\prime} a^{\prime} b^{\prime} a^{\prime}\right)^{\prime}=0 .
\end{aligned}
$$

Proof of (6). Using (II) and (2), we find that

$$
\begin{aligned}
(a, a, b)^{\prime}-a_{b a} & =-(a, b, a)^{\prime}-(a \cdot b a)^{\prime}+(b a)^{\prime} a^{\prime} \\
& =-(a b \cdot a)^{\prime}+(b a)^{\prime} a^{\prime}=-a^{\prime} b^{\prime} a^{\prime}+(b a)^{\prime} a^{\prime}=-a_{b} a^{\prime} .
\end{aligned}
$$

The second part of (6) follows in like manner, while the final part is a consequence of (4).

Proof of (8). We have 


$$
\begin{aligned}
a^{b} r^{\prime}+r^{\prime} a_{b} & =(a b)^{\prime} r^{\prime}+r^{\prime}(a b)^{\prime}-a^{\prime} b^{\prime} r^{\prime}-r^{\prime} b^{\prime} a^{\prime} \\
& =(a b \cdot r+\boldsymbol{r} \cdot a b-a b \cdot r-r b \cdot a)^{\prime} \\
& =(r[a, b]-(\boldsymbol{r}, b, a))^{\prime}=(\boldsymbol{r}[a, b]+(\boldsymbol{r}, a, b))^{\prime},
\end{aligned}
$$

while

$$
\begin{aligned}
r^{\prime} a^{b}+a_{b} r^{\prime} & =r^{\prime}(a b)^{\prime}+(a b)^{\prime} r^{\prime}-r^{\prime} a^{\prime} b^{\prime}-b^{\prime} a^{\prime} r^{\prime} \\
& =(r \cdot a b+a b \cdot r-r a \cdot b-b a \cdot r)^{\prime} \\
& =([a, b] r-(r, a, b))^{\prime} .
\end{aligned}
$$

Proof of (10). Multiply the second equation of (8) by $a^{b}$ on the left and add the resulting equation to the right multiple of the second equation of (8) by $a_{b}$ to obtain, by (9), $a^{b} r^{\prime} a^{b}+a_{b} r^{\prime} a_{b}=a^{b}([a, b] r)^{\prime}$ $+([a, b] r)^{\prime} a_{b}$. Now let $r=[a, b] r$ in the first equation of (8) and use (1) and (2) to obtain (10).

Simplifications when $2 a=0$ for $a \in R$ implies $a=0$. First, (5) is easier to prove because $a^{a b} a_{b}+a^{b} a_{a b}=2 a^{b} a^{\prime} a_{b}=0$ by (3) and (4). The proof of $p q=0$ is also easier since $(p q-q p, p, q)=(p q+q p, p, q)=0$ gives $(q p, p, q)=0=-(p q)^{2}$.

\section{BIBLIOGRAPHY}

1. I. N. Herstein, Jordan homomorphisms, Trans. Amer. Math. Soc. vol. 81 (1956) pp. 331-341.

2. N. Jacobson and C. E. Rickart, Jordan homomorphisms of rings, Trans. Amer. Math. Soc. vol. 69 (1950) pp. 479-502.

3. E. Kleinfeld, Right alternative rings, Proc. Amer. Math. Soc. vol. 4 (1953) pp. 939-944.

4. R. L. San Soucie, Right alternative rings of characteristic two, Proc. Amer. Math. Soc. vol. 6 (1955) pp. 716-719.

5. M. F. Smiley, Jordan homomorphisms onto prime rings, Trans. Amer. Math. Soc. vol. 84 (1957) pp. 426-429.

State UNIVERSity OF Iowa 